\title{
Supporting Information: \\ Direct detection of miR-122 in hepatotoxicity using dynamic chemical labelling overcomes stability and isomiR challenges
}

Barbara López-Longarela ${ }^{1 \#}$, Emma E. Morrison ${ }^{2 \#}$, John D. Tranter ${ }^{2}$, Lianne Chahman-Vos $^{2}$, Jean-François Léonard ${ }^{3}$, Jean-Charles Gautier ${ }^{3}$, Sébastien Laurent ${ }^{4}$, Aude Lartigau ${ }^{3}$, Eric

Boitier $^{3}$, Lucile Sautier ${ }^{4}$, Pedro Carmona-Saez ${ }^{5}$, Jordi Martorell-Marugan ${ }^{5}$, Richard J. Mellanby $^{6}$, Salvatore Pernagallo ${ }^{1}$, Hugh Ilyine ${ }^{1}$, David M. Rissin ${ }^{7}$, David C. Duffy ${ }^{7}$, James W. Dear ${ }^{2 *} \&$ Juan J. Díaz-Mochón ${ }^{1,5^{*}}$

${ }^{1}$ DestiNA Genomics Ltd., Edinburgh, United Kingdom; DestiNA Genomica S.L. Parque Tecnológico Ciencias de la Salud (PTS), Avenida de la Innovación 1, Edificio BIC, Armilla, Granada, Spain.

${ }^{2}$ Pharmacology, Therapeutics and Toxicology, Centre for Cardiovascular Science, University of Edinburgh, The Queen's Medical Research Institute, 47 Little France Crescent, Edinburgh, EH16 4TJ, UK.

${ }^{3}$ Sanofi R\&D, 13 Quai Jules Guesde, 94400 Vitry-sur-Seine, France.

${ }^{4}$ Sanofi R\&D, 371 Rue du Pr. J. Blayac, 34184 Montpellier, France.

${ }^{5}$ Bioinformatics Unit, Centre for Genomics and Oncological Research: Pfizer/University of Granada/Andalusian Regional Government, PTS, Granada, Spain.

${ }^{6}$ The Royal (Dick) School of Veterinary Studies and the Roslin Institute, The Hospital for Small Animals, University of Edinburgh, UK.

${ }^{7}$ Quanterix Corporation, 900 Middlesex Turnpike, Billerica, MA 01821, USA

\section{Supporting Information Table of Contents}

Supporting Text - Methods used in this work for conventional analysis of microRNA, including RNA extraction, PCR, digital droplet PCR and sequencing- Page S-3 to S-5

Supporting Text - Animal models of liver injury used in this work- Page S-6 to S-7

Supporting Text - Design of Stability Study - Page S-8

Supporting Table S-1. Sequences of capture probe and miR-122 target - Page S-9

Supporting Table S-2. Patient demographics from MAPP Study - Page S-10

Supporting Table S-3. Data from rat model of acetaminophen toxicity - Page S-11

Supporting Table S-4. Calibrator concentrations calculated by DCL assay and ddPCR Page S-12

Supporting Table S-5. Clinical details of dogs with liver disease - Page S-13

Supporting Table S-6. miR-122 isomiRs in serum of DILI patients and healthy individuals Page S-14

Supporting Figure S-1. Dynamic chemistry reaction scheme used in this work - Page S-15 
Supporting Figure S-2. Correlation of miR-122 measured by DCL and PCR in serum samples from the MAPP study - Page S-16

Supporting Figure S-3. Correlation between miR-122 concentrations of rat plasma samples calculated by ddPCR and DCL - Page S-17

Supporting Figure S-4. DCL assay quantifies increased circulating miR-122 in dogs - Page S18

Supporting Figure S-5. Heatmaps presenting the serum concentration of miR-122 isomiRs - Page S-19

Supporting Figure S-6. Degradation of miR-122 isomiRs over time - Page S-20

Supporting References. References - Page S-21 


\section{Supporting Text}

Extraction of microRNA from serum samples. microRNA was extracted using a miRNeasy Serum/Plasma kit (Qiagen, Venlo, Netherlands) following the manufacturer's instructions. Total RNA was extracted from $50 \mu \mathrm{L}$ of serum diluted in $150 \mu \mathrm{L}$ nuclease-free water. Briefly, RNA was extracted from the serum by lysis reagent $(1000 \mu \mathrm{L})$ and chloroform $(200 \mu \mathrm{L})$. After centrifugation at $12,000 \times \mathrm{g}$ for $15 \mathrm{~min}$ at $4{ }^{\circ} \mathrm{C}$, up to $600 \mu \mathrm{L}$ of the aqueous phase was transferred to a new tube with $900 \mu \mathrm{L}$ absolute ethanol. RNA was purified on an RNeasy minElute spin column and eluted in $15 \mu \mathrm{L}$ RNase-free water and stored at $-80^{\circ} \mathrm{C}$. Extraction efficiency was monitored by adding $5.6 \times 10^{8}$ copies of synthetic C.elegans miR39 spike-in control after the addition of lysis reagent before the addition of chloroform and phase separation.

Reverse Transcription and Real-Time Polymerase Chain Reaction [RT-PCR]. SYBR Green PCR. The miScript II Reverse Transcription Kit (Qiagen, Venlo, Netherlands) was used to prepare cDNA according to the manufacturer's instructions. Briefly, $2.5 \mu \mathrm{L}$ of RNA eluate was reverse transcribed into cDNA. The synthesised cDNA was then diluted and used in combination with the miScript SYBR Green PCR kit (Qiagen) and a specific miScript assay targeting hsa-miR-122-5p (Hs_miR-122a_1) (Qiagen) for qPCR. qPCR was performed on the LightCycler 480 (Roche, Burgess Hill, UK) system using cycling parameters recommended for miScript assays. All samples were ran in triplicate.

Taqman PCR. RNA eluates were reverse transcribed by following the manufacturer's instructions with the TaqMan MicroRNA Reverse Transcription Kit (Applied Biosystems, Foster City, CA, USA) and TaqMan MicroRNA assay hsa-miR-122 (Assay ID: 002245) (Applied Biosystems). In reverse transcription, $5 \mu \mathrm{L}$ of RNA was transcribed to complementary DNA (cDNA) in a total volume of $15 \mu \mathrm{L}$. Then, $1.33 \mu \mathrm{L}$ of cDNA was used in combination with the TaqMan Universal PCR Master Mix, no AmpErase UNG (Applied Biosystems) and TaqMan probe from the hsa-miR-122 assay (Applied Biosystems) in a total volume of $20 \mu \mathrm{L}$. qPCR was performed on the LightCycler 480 (Roche) system using cycling parameters recommended for TaqMan assays. All samples were ran in triplicate.

For SYBR Green and Taqman PCR, serial dilutions of known standards were made using synthetic miR-122 (syn has-miR-122-5p, 219600, Qiagen). The dilutions were prepared in triplicate, using the same RT and qPCR protocols as described above. The $\mathrm{Ct}$ values were plotted against the logarithm of the concentration, demonstrating a clear linear 
relationship between $\mathrm{Ct}$ value and $\mathrm{Log}$ (conc.). The resultant regression line was used to ascertain the concentration of the unknown sample set.

Droplet-digital PCR (ddPCR). ddPCR was performed on plasma of rats. The extraction of microRNAs was performed with a Qiacube instrument (Qiagen) using $200 \mu \mathrm{L}$ of plasma. The miRNeasy mini kit (QIAGEN, USA) was used with phase lock gel (QuantaBio, USA) and following the last silica gel column elution, $40 \mu \mathrm{L}$ of extracts were collected and stored at $-80{ }^{\circ} \mathrm{C}$ until downstream analysis. The same extraction procedure was used for spiking experiments with a synthetic rno-miR-122-5p in pooled plasma of naïve rats to evaluate extraction yield. After thawing, the reverse transcription of $5 \mu \mathrm{L}$ of extracts was carried out using the TaqMan ${ }^{\mathrm{TM}}$ MicroRNA Reverse Transcription Kit (ThermoFisher, USA) including the TaqMan RT 5 x primer (assay ID: 002245) specific to rno-miR-122-5p; then, the cDNA were stored at $-80^{\circ} \mathrm{C}$. The day of ddPCR experiment, 2 dilutions (1/10 and 1/100) of cDNA were performed and the droplets were generated using the ddPCR ${ }^{\mathrm{TM}}$ Supermix, the miR122$5 \mathrm{p}$ specific TaqMan assay, the droplet generation oil for Probes and the QX200 ${ }^{\mathrm{TM}}$ Droplet Generator (Bio-Rad). The droplets were collected in 96 well plates and run for PCR. A 40 amplification cycle at $56^{\circ} \mathrm{C}$ was performed with the T100 the samples were kept at $+4^{\circ} \mathrm{C}$ until processed the same day by a QX200 Reader (Bio-Rad). The final quantification of miR-122-5p copies was done using the QuantaSoft ${ }^{\mathrm{TM}}$ Analysis Pro software (BIORAD, USA) according to the Poisson law; all samples were evaluated in triplicate.

RNA sequencing. Total RNA was extracted as described above. Standard quality control steps were included to determine total RNA quantity and quality using Agilent 2100 Bioanalyzer with Eukaryote Total RNA Pico Kit and High Sensitivity DNA Assay (Agilent Technologies, Santa Clara, CA). Libraries were prepared using the TruSeq Stranded mRNA Library preparation Kit (Illumina Inc., San Diego, USA), and RNA sequencing was performed on an Illumina NextSeq 500 sequencing platform.

Data Processing and Statistical Analysis. RNA-Seq data analysis was performed using QuickMIRSeq suite. ${ }^{1}$ Briefly, raw sequences were trimmed in order to remove adapter sequences and random nucleotides introduced during the library preparation. Reads shorter than 15 nucleotides (nt) and larger than $28 \mathrm{nt}$ after trimming were discarded to select those sequences more likely to map against miRNAs. Reads were aligned to human reference genome GRCh38. We configured QuickMIRSeq to map not only against reference miRNAs, but also against possible isomiRs with length variations of \pm 4 nucleotides at the $5^{\prime}$ end and \pm 
5 nucleotides at the 3 ' end. We normalized the miRNA counts by reads per million (RPM) method in order to correct for differences in sequencing depth. We discarded low-expressed miRNAs (less than 50 RPM in at least 6 samples). We used pheatmap R package for generating heatmaps. Data are presented as median (IQR) unless specifically indicated. Statistical analysis was as described in the results. 


\section{Supporting Text}

Rat model of drug-induced liver injury. An investigative rat toxicology study was conducted. In brief, purpose-bred male Sprague Dawley rats (age at initiation of dosing: 9-10 weeks) were supplied by Charles River Laboratories (Calco, Italy) and acclimatized for 14 days before the start of the experiment. The animals were housed in a facility accredited by the Association for Assessment and Accreditation of Laboratory Animal Care International. The study and all procedures were in accordance with the Directive 2010/63/EU of the European parliament and the related French transposition texts, and were approved by the Sanofi Animal Care and Use Committee. The day of arrival, the animals were randomly allocated to the vehicle group (6 rats) or the compound-dosed group (6 rats). The 6 animals from the compound-dosed group were given a single oral administration of acetaminophen in the vehicle (Methylcellulose, Tween 80, Water at 0.5, 0.1 and 99.4\%, respectively) at the dose level of $1500 \mathrm{mg} / \mathrm{kg}$, and the control group received the respective vehicle. Food was withheld overnight before necropsy. $24 \mathrm{~h}$ after single dosing, animals were anesthetised with isoflurane and blood samples were collected at the abdominal aorta for clinical chemistry analyses $(900 \mu \mathrm{L}$ in heparin lithium vacutainer tubes) and miR-122 measurements $(400 \mu \mathrm{L}$ in EDTA K2 vacutainer tubes frozen and stored at $\left.-80{ }^{\circ} \mathrm{C}\right)$. Animals were then euthanized by exsanguination and a full necropsy was performed with macroscopic examination of the thoracic and abdominal cavities and organs. The liver, heart, kidneys and brain were weighed. The liver (left and right lateral lobes as well as median lobe), heart, kidneys and quadriceps femoris muscle were collected for histopathological evaluation, fixed in 10\% neutral buffered formalin, and paraffin-embedded within $48 \mathrm{~h}$. Sections were then stained with haematoxylin and eosin (H\&E) and examined by two board-certified veterinarian pathologists. Microscopic findings were classified according to the INHAND (International Harmonization of Nomenclature and Diagnostic Criteria) nomenclature system ${ }^{2}$ and graded on a scale of $0-5$, where $0=$ no change, $1=$ minimal, $2=$ slight, $3=$ moderate, $4=$ marked and $5=$ severe. Plasma alanine transaminase (ALT) and glutamate dehydrogenase (GLDH) activites were measured on a Roche Cobas 6000 c501 on an automated clinical chemistry analyser using reagents purchased from Roche Diagnostics.

Treated animals had increased plasma ALT activity $24 \mathrm{~h}$ post-dose $($ mean $=518.8$ $\mathrm{U} / \mathrm{L}$, s.d. $=754.2)$ when compared to controls $($ mean $=35.8 \mathrm{U} / \mathrm{L}, \mathrm{s} . \mathrm{d} .=27.4, \mathrm{p}<0.05$, MannWhitney test), and increased plasma GLDH activity (treated: mean $=207.7 \mathrm{U} / \mathrm{L}$, s.d. $=176.0$; controls: mean $=3.7 \mathrm{U} / \mathrm{L}$, s.d. $=1.9, \mathrm{p}<0.05$, Mann-Whitney test). At terminal sacrifice, the 
mean absolute liver weight from the treated group was $16 \%$ higher $(\mathrm{p}<0.05$, Mann-Whitney test) when compared with control animals. Microscopically, the liver from acetaminophentreated rats showed minimal to marked hepatocellular centrilobular apoptosis and/or necrosis, together with minimal to moderate multifocal neutrophilic and/or mononuclear cell inflammatory infiltration. ${ }^{1}$ There were no compound-related microscopic changes in the heart, kidneys and skeletal muscle from treated rats.

Dogs with clinical liver disease. All dogs were recruited to this study at the Royal (Dick) School of Veterinary Studies (R(D)SVS), Edinburgh, UK. Healthy dogs presenting to the R[D]SVS General Practice for routine annual vaccination, who had a normal history and clinical examination, were invited to have a serum biochemical health screen that included measurement of ALT activity. Dogs that had a diagnostic assessment that included histopathological examination of a liver biopsy and serum ALT activity measurement leading to definitive diagnosis of a primary liver disorder were also enrolled into the study. The histopathological diagnosis was classified according to WSAVA (World Small Animal Veterinary Association) criteria by a board certified pathologist. The study was approved by The University of Edinburgh Veterinary Ethics Research Committee. 


\section{Supporting Text}

\section{$\underline{\text { Stabilization study design }}$}

$\underline{\operatorname{Day} 0(T 0)}$

\section{PCR analysis:}

a) Frozen serum samples were thawed and aliquoted for testing at T0 (day 0), T1 (day 1), T7 (day 7), T14 (day 14) and T28 (day 28). For each time point three replicates were used. (30 $\mu \mathrm{L}$ serum/replicate).

b) RNA extraction and PCR for T0 samples was performed using SYBR-green based PCR.

c) The remaining PCR samples were stored at room temperature.

\section{DCL analysis:}

a) Frozen serum samples were thawed and aliquoted for testing at T0, T1, T7, T14 and T28. For each time point three replicates were used. (30 $\mu \mathrm{L}$ serum/replicate).

b) For time point $\mathrm{T} 0$ three replicates were used (30 $\mu \mathrm{L}$ serum/replicate) containing serum + beads + Stabiltech buffer. T0 samples were analysed along with a calibration curve.

c) For time points T1, T7, T14, T28 there were two groups of samples:

Group 1. Serum + beads + Stabiltech Buffer at T0. Analysis was performed at performed at $\mathrm{T} 1, \mathrm{~T} 7, \mathrm{~T} 14$ or $\mathrm{T} 28$.

Group 2. Serum kept at room temperature until addition of beads + Stabiltech buffer at T1, T7, T14 or T28. Analysis was performed at T1, T7, T14 or T28.

Day 1 (T1), Day 7 (T7), Day 14 (T14) and Day 28 (T28).

PCR analysis:

RNA extraction and SYBR-green based PCR was performed for room temperature stored T1, T7, T14 or T28 samples.

\section{DCL analysis:}

a) Beads + lysis buffer were added to the samples from Group 2 (T1, T7, T14 or T28). Calibration curves were prepared at the same time as the combining of beads plus lysis buffer with the samples.

b) Samples from Groups 1 and 2 (T1, T7, T14 or T28) were analysed along with the corresponding calibration curves. 


\begin{tabular}{|c|c|c|}
\hline ID & Name & Peptide with abasic position ( $\left.\mathrm{N}^{\prime}-\mathrm{C}^{\prime}\right)$ \\
\hline 1 & $\begin{array}{l}\text { Capture probe } \\
\text { (DGL 122_4.3) }\end{array}$ & $\mathrm{xx}-\mathrm{CACCATT} * \mathrm{GT} * \mathrm{GL} * \mathrm{AC}^{*} \mathrm{ACT} * \mathrm{CCA}$ \\
\hline & & miRNA-122-5p sequence (5' -3 ') \\
\hline 2 & Target miR-122 & UGGAGUGUGACAAUGGUGUUUG \\
\hline
\end{tabular}

Supporting Table S-1. Sequences of capture probe (1) and miR-122 target (2).

Key: $\mathrm{xx}=$ amino-PEG linker; $\mathrm{T}^{*}=$ thymidine containing a propanoic acid side chain at the gamma position; $\mathrm{C}^{*}=$ cytosine containing a propanoic acid side chain at the gamma position; *GL* = abasic "blank" monomer presenting a secondary amine and containing a propanoic acid side chain at the gamma position; the italicized bases in $\mathbf{2}$ form a duplex with the capture probe, and $\mathrm{G}$ at position 9 (bold) is opposite the "blank" monomer and binds to the aldehydemodified cytosine. The mature sequence of miRNA-122 is 22 bases long. Our probe targets 18 bases out of the 22 , leaving out the 4 bases at the 3 '-end. 


\begin{tabular}{|c|c|}
\hline Number & 192 \\
\hline Sex (number M:F) & $66: 126$ \\
\hline Age (years) & $28(19-39)$ \\
\hline Amount of acetaminophen ingested $(\mathrm{g})$ & $17(9-21)$ \\
\hline Time from ingestion to first blood sample (h) & $4(4-8)$ \\
\hline Admission acetaminophen concentration $(\mathrm{mg} / \mathrm{L})$ & $111(62-150)$ \\
\hline Admission ALT $(\mathrm{U} / \mathrm{l})$ & $16(12-24)$ \\
\hline Admission ALP $(\mathrm{U} / \mathrm{l})$ & $69(46-78)$ \\
\hline Admission INR & $1(1-1.1)$ \\
\hline Admission serum creatinine $(\mu \mathrm{mol} / \mathrm{L})$ & $59(50-68)$ \\
\hline Number with admission ALT $<\mathrm{ULN}$ & 175 \\
\hline Number with admission ALT $>100$ & 3 \\
\hline Number with admission ALT $>1000$ & 0 \\
\hline Number with Peak ALT $>100$ & 18 \\
\hline Number with Peak ALT $>1000$ & 9 \\
\hline
\end{tabular}

Supporting Table S-2. Patient demographics from the MAPP Study. ALT = serum alanine transaminase activity. $\mathrm{ALP}=$ serum alkaline phosphatase activity. $\mathrm{INR}=$ international normalised ratio. Data are presented as median (IQR) for continuous variables. 


\begin{tabular}{|c|c|c|c|c|c|c|c|c|c|}
\hline$\underset{N^{\circ}}{\text { Animal }}$ & Group & $\begin{array}{c}\text { Dose } \\
(\mathrm{mg} / \mathrm{kg})\end{array}$ & $\begin{array}{c}\text { Hepatocyte } \\
\text { Necrosis } \\
\text { Score }\end{array}$ & $\begin{array}{c}\text { Hepatocyt } \\
\text { e } \\
\text { Apoptosis } \\
\text { Score }\end{array}$ & $\begin{array}{c}\text { ALT } \\
(\mathbf{U} / \mathbf{L})\end{array}$ & $\begin{array}{c}\text { GLDH } \\
(\mathbf{U} / \mathbf{L})\end{array}$ & $\begin{array}{c}\text { DCL } \\
\text { miR-122 } \\
(\mathbf{p M})\end{array}$ & $\begin{array}{c}\text { DCL } \\
\text { miR-122 } \\
(\operatorname{copy} / \mu L \\
)\end{array}$ & $\begin{array}{c}\text { ddPCR } \\
\text { miR-122 } \\
(\operatorname{copy} / \mu \mathrm{L})\end{array}$ \\
\hline 61 & Control & 0 & 0 & 0 & 30 & $<\mathrm{LLOQ}$ & $<$ LLOQ & $<$ LLOQ & $1.2 \mathrm{E}+03$ \\
\hline 62 & Control & 0 & 0 & 0 & 23 & $<\mathrm{LLOQ}$ & 14 & $8.4 \mathrm{E}+06$ & $1.2 \mathrm{E}+03$ \\
\hline 63 & Control & 0 & 0 & 0 & 91 & $<\mathrm{LLOQ}$ & $<$ LLOQ & $<$ LLOQ & $4.5 \mathrm{E}+02$ \\
\hline 64 & Control & 0 & 0 & 0 & 21 & $<$ LLOQ & $<$ LLOQ & $<$ LLOQ & $1.5 \mathrm{E}+03$ \\
\hline 65 & Control & 0 & 0 & 0 & 20 & 6 & $<$ LLOQ & $<$ LLOQ & $3.3 \mathrm{E}+03$ \\
\hline 66 & Control & 0 & 0 & 0 & 30 & 6.4 & 18 & $1.1 \mathrm{E}+07$ & $5.6 \mathrm{E}+03$ \\
\hline 67 & $\begin{array}{c}\text { Acetamino } \\
\text { phen }\end{array}$ & 1500 & 1 & 4 & 674 & 119.3 & 2705 & $1.6 \mathrm{E}+09$ & $2.0 \mathrm{E}+05$ \\
\hline 68 & $\begin{array}{l}\text { Acetamino } \\
\text { phen }\end{array}$ & 1500 & 0 & 2 & 76 & 62.7 & 435 & $2.6 \mathrm{E}+08$ & $5.7 \mathrm{E}+04$ \\
\hline 69 & $\begin{array}{c}\text { Acetamino } \\
\text { phen }\end{array}$ & 1500 & 0 & 1 & 41 & 5.5 & 32 & $1.9 \mathrm{E}+07$ & $1.1 \mathrm{E}+04$ \\
\hline 70 & $\begin{array}{c}\text { Acetamino } \\
\text { phen }\end{array}$ & 1500 & 4 & 3 & 1985 & 431.9 & 5345 & $3.2 \mathrm{E}+09$ & $1.4 \mathrm{E}+06$ \\
\hline 71 & $\begin{array}{c}\text { Acetamino } \\
\text { phen }\end{array}$ & 1500 & 0 & 2 & 184 & 234.1 & 594 & $3.6 \mathrm{E}+08$ & $2.8 \mathrm{E}+05$ \\
\hline 72 & $\begin{array}{c}\text { Acetamino } \\
\text { phen }\end{array}$ & 1500 & 0 & 1 & 153 & 392.4 & 1056 & $6.4 \mathrm{E}+08$ & $4.5 \mathrm{E}+05$ \\
\hline
\end{tabular}

Supporting Table S-3. Data from rats treated with a single dose of acetaminophen (1500 $\mathrm{mg} / \mathrm{kg}$ oral) or vehicle control. Plasma miR-122 concentrations were measured by both DCL and ddPCR. Hepatocellular necrosis and apoptosis were scored on H\&E-stained sections of the right lateral liver lobe as described in the methods. Hepatocellular apoptosis was further confirmed by immunohistochemical detection of activated caspase 3 on formalin fixed, paraffin-embedded sections of the liver. LLOQ $=$ lower limit of quantification. 


\begin{tabular}{|c|c|c|c|c|}
\hline \multicolumn{3}{|c|}{ Theoretical titer } & & \\
\hline $\begin{array}{c}\text { Study } \\
\text { ID }\end{array}$ & 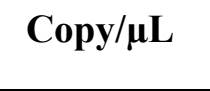 & $\mathbf{p M}$ & & \\
\hline $\mathrm{C}-1$ & 6022000 & 10 & & \\
\hline $\mathrm{C}-2$ & 60220000 & 100 & & \\
\hline $\mathrm{C}-3$ & 301100000 & 500 & & \\
\hline \multicolumn{5}{|c|}{ ddPCR evaluation } \\
\hline $\begin{array}{c}\text { Study } \\
\text { ID }\end{array}$ & 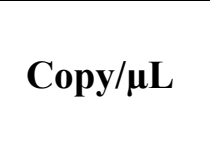 & $\mathbf{p M}$ & $\begin{array}{l}\text { Fold difference } \\
\text { expected vs } \\
\text { obtained }\end{array}$ & CV (\%) \\
\hline $\mathrm{C}-1$ & 2918 & 0.0048 & 2083.3 & 4.2 \\
\hline $\mathrm{C}-2$ & 11517 & 0.0191 & 5235.6 & 7.2 \\
\hline $\mathrm{C}-3$ & 28746 & 0.0477 & 10482.1 & 3.9 \\
\hline \multicolumn{5}{|c|}{ DCL evaluation } \\
\hline $\begin{array}{c}\text { Study } \\
\text { ID }\end{array}$ & 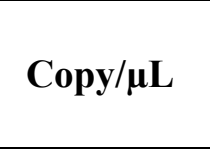 & $\mathbf{p M}$ & $\begin{array}{c}\text { Fold difference } \\
\text { expected vs } \\
\text { obtained }\end{array}$ & CV (\%) \\
\hline $\mathrm{C}-1$ & 4145513 & 6.883 & 1.45 & 16.8 \\
\hline $\mathrm{C}-2$ & 43032534 & 71.43 & 1.39 & 2.2 \\
\hline $\mathrm{C}-3$ & 212409632 & 352.66 & 1.41 & 8.0 \\
\hline
\end{tabular}

Supporting Table S-4. Calibrator concentrations of miR-122 spiked into healthy rat plasma obtained by both the DCL assay and ddPCR. Fold differences between expected calibrator concentrations and those obtained are expressed as the ratio between expected vs obtained values. $\mathrm{CV}=$ coefficient of variation. 


\begin{tabular}{|l|l|l|l|}
\hline Breed & Age (Yrs) & Diagnosis & ALT (U/L) \\
\hline Havanese & 8 & cholestasis & 656 \\
\hline English Springer Spaniel & 11 & chronic hepatitis & 193 \\
\hline Scottish Terrier & 9 & lymphoma & 233 \\
\hline Flat Coated Retriever & 9 & adenoma & 776 \\
\hline English Pointer & 6 & granuloma, hepatitis & 1544 \\
\hline Cairn Terrier & 11 & hepatocellular carcinoma & 182 \\
\hline Labrador & 8 & chronic-active hepatitis & 295 \\
\hline West Highland White Terrier & 2 & none & 55 \\
\hline Old English Sheepdog & 5 & hepatitis, chronic & 1235 \\
\hline Labrador Cross & 13 & cholangiocellular carcinoma & 46 \\
\hline Bearded Collie & 13 & hepatopathy & 33 \\
\hline Labrador & 8 months & lobular dissecting hepatitis & 201 \\
\hline
\end{tabular}

Supporting Table S-5. Clinical details of dogs with liver disease. 


\begin{tabular}{|l|l|l|l|l|l|l|}
\hline & $\begin{array}{l}\text { Healthy } \\
\text { sample 1 } \\
\text { (RPM) }\end{array}$ & $\begin{array}{l}\text { Healthy } \\
\text { sample } \\
\text { (RPM) }\end{array}$ & $\begin{array}{l}\text { Healthy } \\
\text { sample } \\
\text { IsomiR }\end{array}$ & $\begin{array}{l}\text { DILI } \\
\text { (Rample 1 } \\
\text { (RPM) }\end{array}$ & $\begin{array}{l}\text { DILI } \\
\text { sample 2 } \\
\text { (RPM) }\end{array}$ & $\begin{array}{l}\text { DILI } \\
\text { sample 3 } \\
\text { (RPM) }\end{array}$ \\
\hline hsa-miR-122-5p_0_-1 & 187.85 & 366.56 & 274.7 & 332446.44 & 299487.41 & 311780.78 \\
\hline hsa-miR-122-5p_0_0 & 95.89 & 151.16 & 137.72 & 142689.37 & 138157.28 & 137990.22 \\
\hline hsa-miR-122-5p_0_1 & 89.56 & 68.4 & 81.62 & 42105.79 & 44347.71 & 44885.22 \\
\hline hsa-miR-122-5p_0_-3 & 2.66 & 14.22 & 6.83 & 9849.26 & 9791.23 & 9543.85 \\
\hline hsa-miR-122-5p_0_-2 & 1.9 & 14.62 & 4.01 & 8384.43 & 8419.18 & 8315.69 \\
\hline hsa-miR-122-5p_-1_-1 & 1.27 & 3.89 & 3.86 & 6194.05 & 5296.08 & 5674.95 \\
\hline hsa-miR-122-5p_-1_0 & 1.14 & 1.21 & 1.04 & 2698.76 & 2540.07 & 2458.8 \\
\hline hsa-miR-122-5p_0_-5 & 1.39 & 1.21 & 1.63 & 2173.99 & 2008.81 & 1959.81 \\
\hline hsa-miR-122-5p_0_-6 & 0.25 & 7.24 & 9.2 & 2171.1 & 2149.94 & 2041.44 \\
\hline hsa-miR-122-5p_0_-4 & 1.39 & 0.94 & 1.48 & 1977.07 & 1897.31 & 1951.41 \\
\hline hsa-miR-122-5p_-2_-1 & 0.38 & 0.67 & 1.34 & 1367.41 & 1166.56 & 1192.23 \\
\hline hsa-miR-122-5p_-4_-1 & 1.01 & 0.54 & 1.19 & 1109.43 & 1063.8 & 1002.78 \\
\hline hsa-miR-122-5p_-1_1 & 0.38 & 0.13 & 0.15 & 1016.45 & 1072.23 & 1148.35 \\
\hline hsa-miR-122-5p_1_-1 & 0.25 & 0.4 & 0.45 & 910.26 & 830.8 & 849.17 \\
\hline hsa-miR-122-5p_-3_-1 & 0.25 & 0.54 & 0.15 & 777.5 & 658.08 & 698.09 \\
\hline hsa-miR-122-5p_-2_0 & 0.13 & 0.13 & 0.15 & 455.45 & 402.66 & 400.06 \\
\hline hsa-miR-122-5p_-4_0 & 0.13 & 0.13 & 0.15 & 419 & 345.62 & 375.48 \\
\hline hsa-miR-122-5p_1_0 & 0 & 0.27 & 0.15 & 359.01 & 337.09 & 333.9 \\
\hline hsa-miR-122-5p_-3_0 & 0.13 & 0 & 0.3 & 269.64 & 210.18 & 238.94 \\
\hline hsa-miR-122-5p_-1_-3 & 0 & 0.13 & 0.3 & 176.78 & 206.82 & 170.2 \\
\hline hsa-miR-122-5p_0_-7 & 0.13 & 0 & 0 & 116.41 & 121.28 & 100.75 \\
\hline hsa-miR-122-5p_0_-8 & 0 & 0.27 & 0 & 89.5 & 81.15 & 85.5 \\
\hline hsa-miR-122-5p_0_-9 & 0 & 0 & 16.62 & 72.08 & 79.82 & 77.27 \\
\hline hsa-miR-122-5p_-1_-6 & 0 & 0 & 0.15 & 46.24 & 53.86 & 39.21 \\
\hline hsa-miR-122-5p_0_-10 & 0 & 0 & 0 & 29.42 & 35.9 & 30.68 \\
\hline
\end{tabular}

Supporting Table S-6. miR-122 isomiRs in serum of DILI patients and healthy individuals. $\mathrm{RPM}=$ reads per million. 


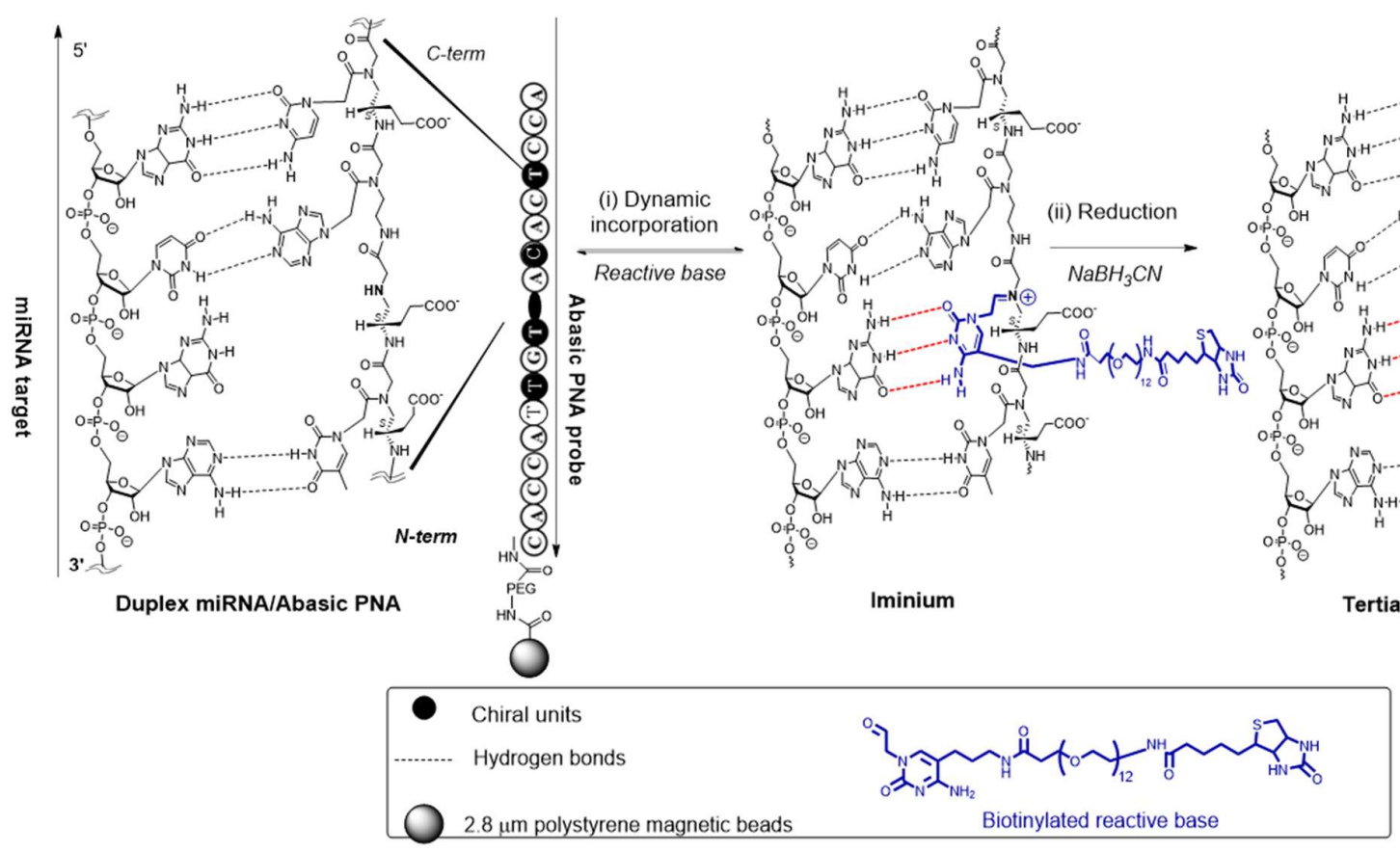

Supporting Figure S-1. Dynamic chemistry labelling of biotin into an immobilized abasic PNA probe on a superparamagnetic bead. 

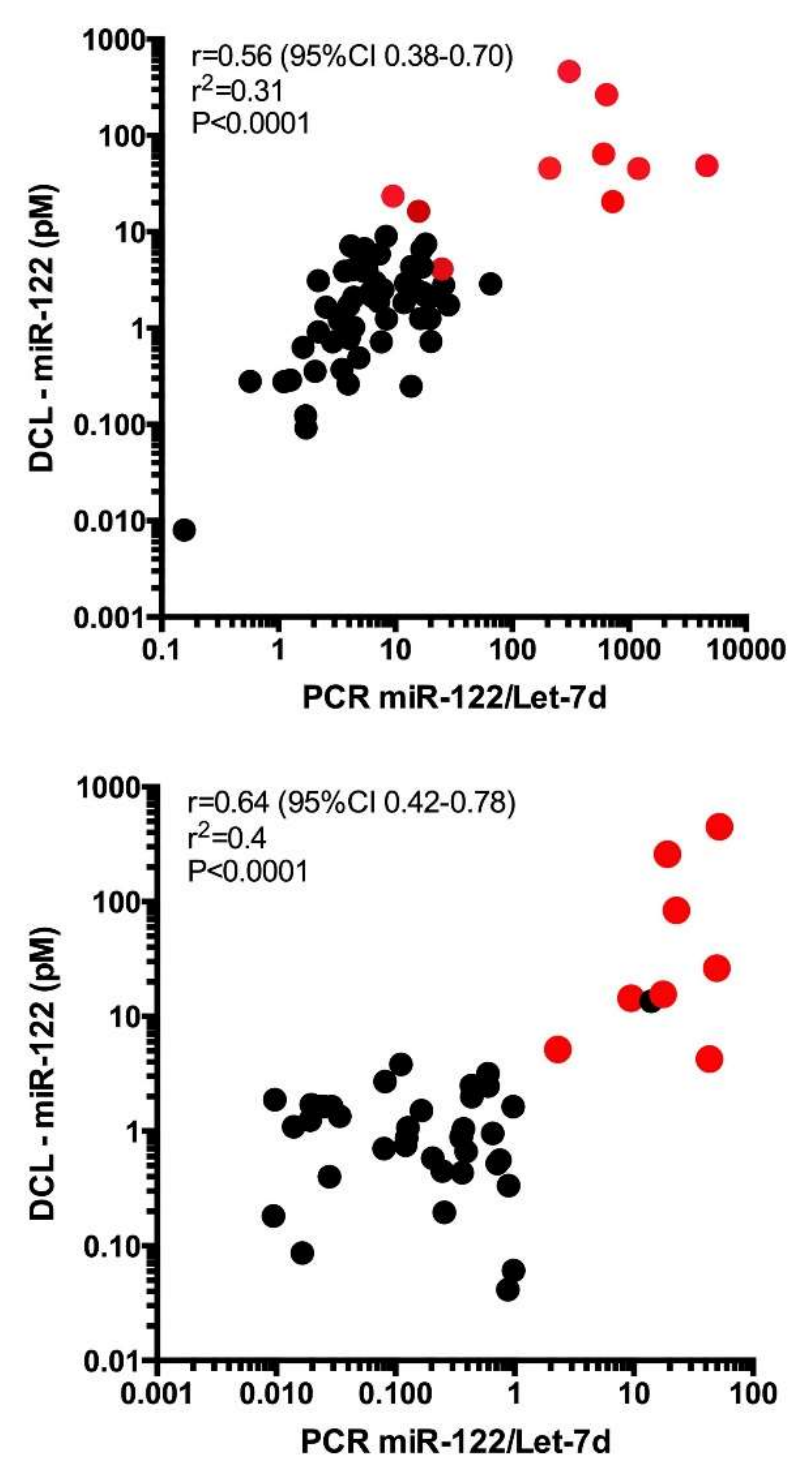

Supporting Figure S-2. miR-122 was measured by DCL and PCR in serum samples from the MAPP study. The PCR data is from the original analysis with normalisation to the microRNA Let7d. The two panels present data from patients recruited at two UK hospitals, St Thomas' Hospital London and Aberdeen Royal Infirmary. Pearson correlation was performed to explore the relationship between the two techniques. Red points represent study participants who developed acute liver injury (ALT $>100 \mathrm{U} / \mathrm{L})$. 


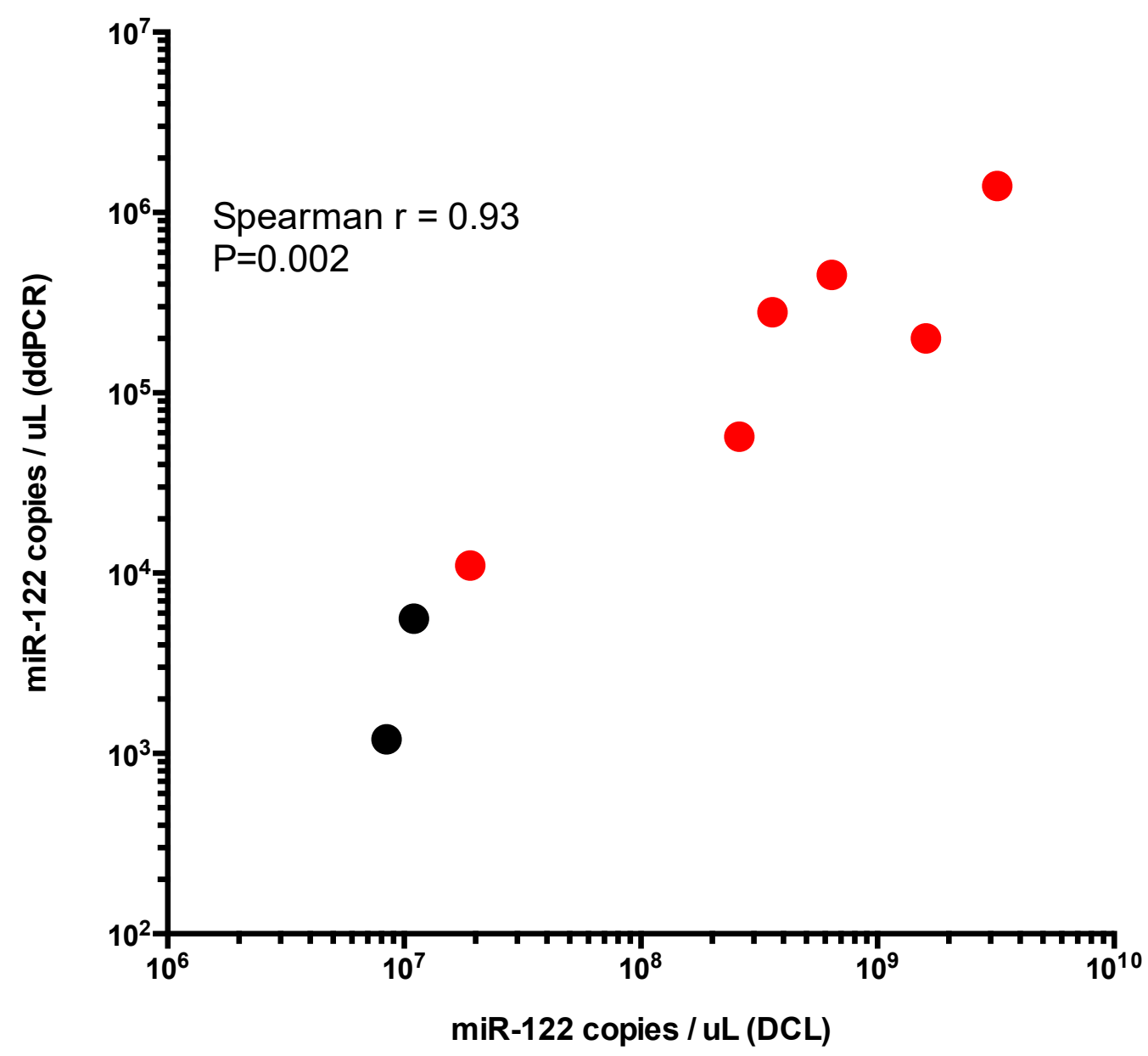

Supporting Figure S-3. Correlation between miR-122 concentrations of rat plasma samples (see Supporting Table 2) calculated by ddPCR and DCL. Red dots are rats treated with acetaminophen. The two black dots correspond to the 2 control rats with values of DCL above LLOQ. The values of ddPCR in the 4 control rats with DCL values below the LLOQ (not represented in the graph) were between $4.5 \mathrm{E}+02$ and $3.3 \mathrm{E}+03$ copies per microliter. 


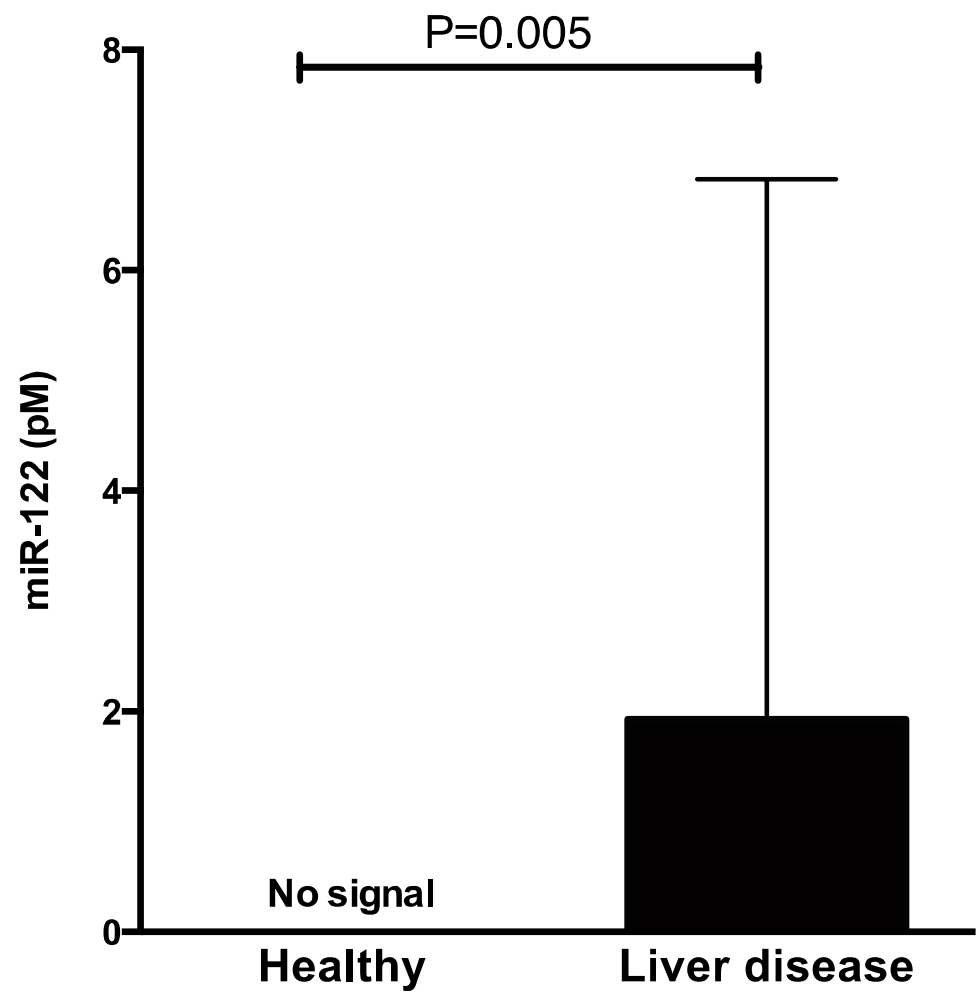

Supporting Figure S-4. DCL assay quantifies increased circulating miR-122 in dogs. miR-122 was increased in dogs with clinical liver disease compared with healthy dogs ( $\mathrm{N}=12$ per group). Graphs present median values, bars present the IQR. P values calculated by Mann-Whitney test. 
A. Serum from health and DILI at TO

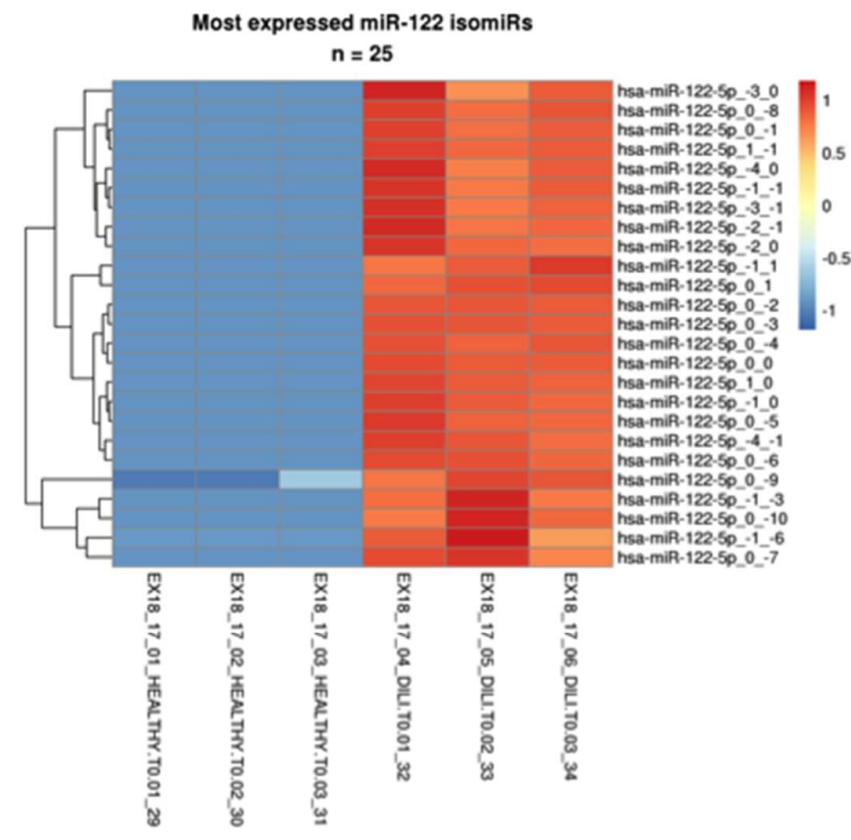

\section{B. IsomiR concentrations over time}

DILI serum

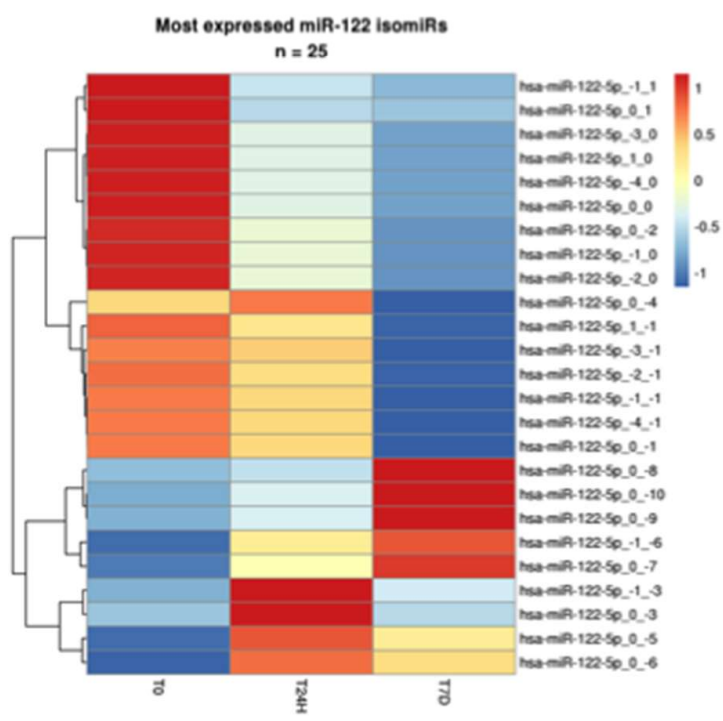

C. IsomiR concentrations over time Healthy serum

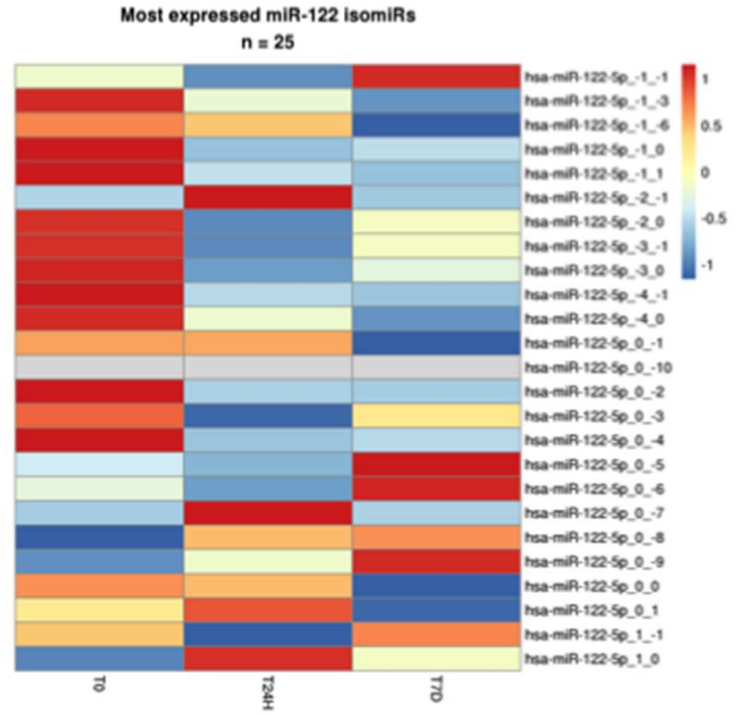

Supporting Figure S-5. Heatmaps presenting the serum concentration of miR-122 isomiRs. A. Small RNA sequencing was performed on normal and DILI serum (ALT > $1000 \mathrm{U} / \mathrm{L}$ ). Multiple isomiRs of miR-122 were increased with DILI when there was no delay in processing (T0) ( $\mathrm{N}=3$ per group). B. DILI serum was left at room temperature for 1 and 7 days (T24H and T7D). The change in isomiRs is presented. C. Healthy serum was left at room temperature for 1 and 7 days (T24H and T7D). The change in isomiRs is presented. 

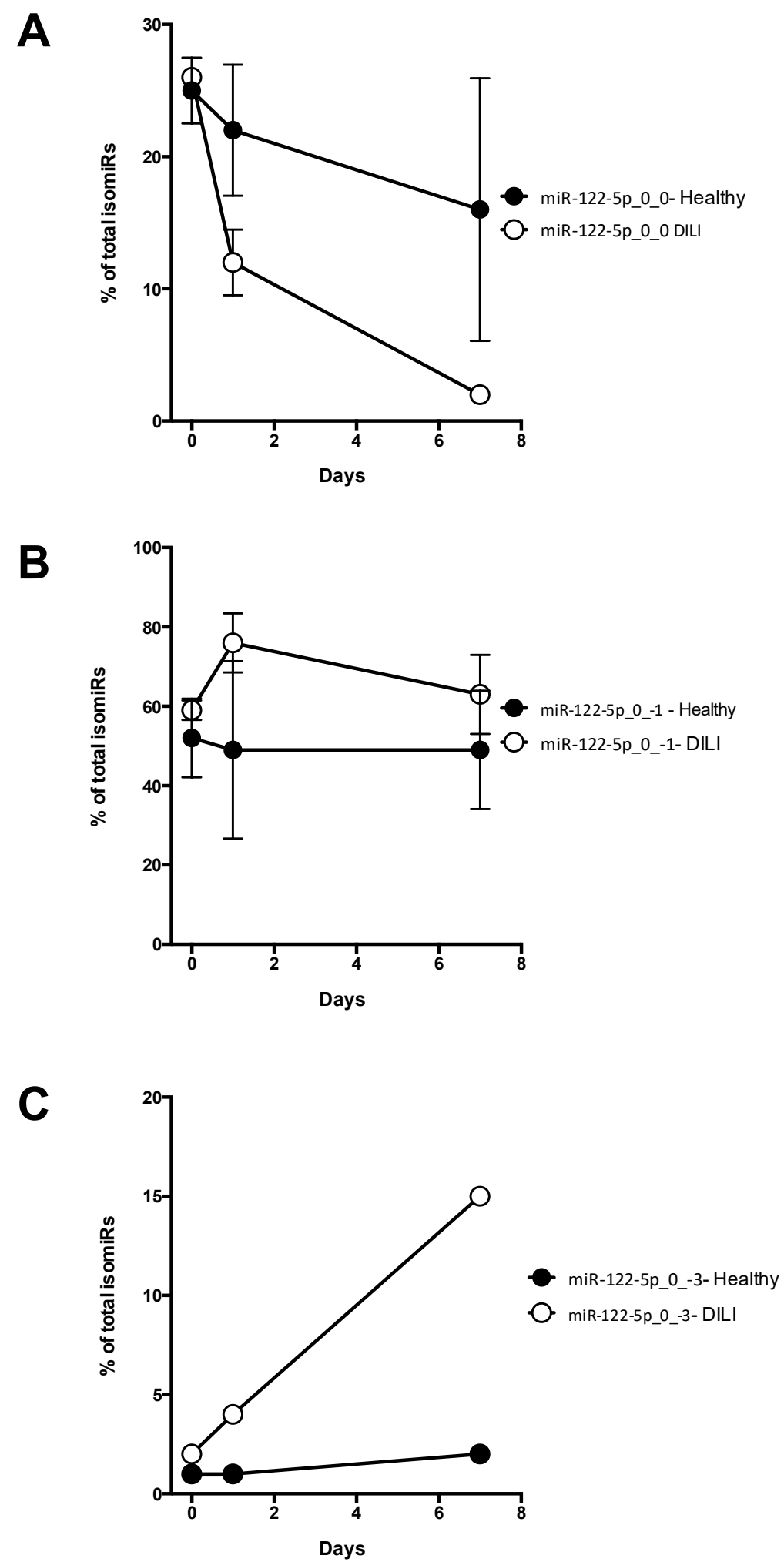

Supporting Figure S-6. RNA sequencing demonstrated that the canonical form of miR-122 (miR-122-5p_0_0) (A) degrades over time with a concurrent increase in shorter isomiRs (miR-122-5p_0_-1 and miR-122-5p_0_-3) (B and C, respectively). The degradation is enhanced with $\bar{D}$ ILI. Data are represented as mean and $95 \% \mathrm{CI}$ 


\section{Supporting References}

1. Zhao, S.; Gordon, W.; Du, S.; Zhang, C.; He, W.; Xi, L.; Mathur, S.; Agostino, M.; Paradis, T.; von Schack, D.; Vincent, M.; Zhang, B., QuickMIRSeq: a pipeline for quick and accurate quantification of both known miRNAs and isomiRs by jointly processing multiple samples from microRNA sequencing. BMC Bioinformatics 2017, 18 (1), 180.

2. Thoolen, B.; Maronpot, R. R.; Harada, T.; Nyska, A.; Rousseaux, C.; Nolte, T.; Malarkey, D. E.; Kaufmann, W.; Kuttler, K.; Deschl, U.; Nakae, D.; Gregson, R.; Vinlove, M. P.; Brix, A. E.; Singh, B.; Belpoggi, F.; Ward, J. M., Proliferative and nonproliferative lesions of the rat and mouse hepatobiliary system. Toxicol Pathol 2010, 38 (7 Suppl), 5S-81S. 